\title{
Clinical and cost-effectiveness of absorbent dressings in the treatment of highly exuding VLUs
}

- Objective: To estimate the clinical effectiveness and cost effectiveness of using a sodium
carboxymethylcellulose dressing (CMC [Aquacel]) and four superabsorbent dressings (DryMax Extra [DM], Flivasorb [F], Kerramax [K] and sachet $\mathrm{S}[\mathrm{S}]$ ) in the treatment of highly exuding chronic venous leg ulcers (VLUs) in the UK, from the perspective of the National Health Service (NHS).

- Method: A decision model was constructed depicting the patient pathways and associated management of a cohort of 439 patients with highly exuding chronic VLUs of $\geq 3$ months of age. The model was based on the case records of a cohort of matched patients from The Health Improvement Network (THIN) database (a nationally representative database of patients registered with general practitioners (GPs) in the UK) who were treated with one of the five dressings. The model estimated the costs and outcomes of patient management over 6 months and the relative cost-effectiveness of using each dressing.

- Results: Patients' mean age was 73.1 years, and $46 \%$ were female. Between $39 \%$ and $56 \%$ of VLUs healed by 6 months. CMC-treated wounds that remained unhealed increased in size by $43 \%$ over the study period, whereas unhealed wounds treated with the other dressings decreased in size by a mean $34 \%$. Consequently, CMC was excluded from the cost-effectiveness analysis. The 6-monthly NHS cost of managing a VLU with $S$ was $£ 3700$ per patient, which was $15-28 \%$ lower than the cost of managing patients with the other three superabsorbents. Additonally, use of $S$ improved patients' health status to a greater extent than the other three superabsorbents, since S-treated patients accrued $0.3-3 \%$ more QALYs. Starting treatment with $\mathrm{S}$ was the preferred strategy followed by DM, K and $\mathrm{F}$ in that order. - Conclusion: Within the limitations of the data set, $S$ affords the NHS a cost-effective treatment for managing highly exuding chronic VLUs of $\geq 3$ months of age, compared with DM, F, K and CMC.

- Declaration of interest: This study was funded with an unrestricted research grant from Sorbion GmbH \& Co. KG, Senden, Germany, manufacturers of sachet S. However, Sorbion did not have any control of the methodology, conduct, results or conclusion of this study, or editorial involvement in this manuscript. The authors have no other conflicts of interest that are directly relevant to the content of this manuscript, which remains their sole responsibility

absorbent dressing, cost-effectiveness, QALY, venous leg ulcers, United Kingdom

xudate is fluid that filters from the circulatory system as a result of inflammatory processes, and in acute wounds maintains a moist environment and promotes healing. ${ }^{1}$ In chronic wounds, exudate contains a high level of proteases, which can be a significant factor in delaying wound healing. ${ }^{2}$ A low level of exudate can result in wound dessication and this can inhibit the healing process. ${ }^{3}$ However, an unmanaged high level of exudate can cause peri-wound skin damage, ${ }^{4}$ and may be harmful to the wound bed itself. The optimal level of exudate necessary for healthy uncomplicated wound healing is unknown. ${ }^{5}$

Exudate becomes a problem when a dressing leaks, a high frequency of dressing change is required, there are peri-wound changes, such as maceration or skin stripping, patients experience discomfort or pain, or there is fluid and electrolyte imbalance owing to protein loss. ${ }^{2}$ Effective exudate management using an appropriate dressing is critical.

Successful wound management requires a flexible approach to the selection and use of dressings, based on an understanding of the healing process and knowledge of the properties of the various dressings available. ${ }^{2}$ Many factors influence dressing selection, but it should be based upon holistic assessment, including physical examination of the patient, past medical history and wound history. ${ }^{6}$ Superabsorbent dressings have been designed to manage moderate to highly exuding wounds. The dressings contain the superabsorbent particle sodium polyacrylate, with high absorbency. In the UK, several superabsorbents are available on the market, including:

- DryMax Extra (DM; Absorbest AB)

- KerraMax (K; Crawford Healthcare Ltd.)

- Flivasorb (F; Lohmann \& Raushcer GmbH \& Co.) - sachet S (S; Sorbion GmbH \& Co. KG).
M. Panca,' MSc; K. Cutting, ${ }^{2} \mathrm{MN}, \mathrm{DipN}$; J.F. Guest, ${ }^{1,3} \mathrm{PhD}$; I Catalyst Health Economics Consultants Middlesex, UK:

2 Buckinghamshire New University, Uxbridge, UK and Perfectus Medical, Daresbury, UK;

3 School of Biomedical Sciences, King's College, London, UK.

Email: julian.guest@ catalyst-health.co.uk 
- Sodium carboxymethylcellulose (CMC [Aquacel]; Convatec Inc.) is not a superabsorbent, but is used extensively to treat highly exuding wounds in the UK.

Therefore, the objective of this study was to estimate the relative clinical and cost-effectiveness of using DM, K, F, S and CMC in the treatment of highly exuding chronic venous leg ulcers (VLUs) in the UK, from the perspective of the NHS.

\section{Method}

The Health Improvement Network (THIN) database contains computerised information on more than 9 million anonymised patients, entered by general practitioners (GPs), from 500 practices across the UK. ${ }^{7}$ General practices across the UK using Vision Practice Management Software are invited to participate in the database, and are self-selecting. The data within THIN has been shown to be representative of the UK population, in terms of demographics and disease distribution. ${ }^{8,9}$

The computerised information in the THIN database includes patients' demographics, details from GP consultations, specialist referrals, nurse and other clinician visits, hospital admissions, diagnostic and therapeutic procedures, laboratory tests and prescriptions issued by GPs, which are directly generated by the general practice's IT system. Hence, the information contained in the THIN database reflects real clinical practice, as it is based on actual patient records. Moreover, GPs are the gatekeepers to health care in the UK, and patients' entire medical history is theoretically stored in their primary care record.

Specific diagnoses are recorded using Read codes. Read codes are a coded thesaurus of clinical terms that are used by clinicians in the UK to record patient findings and procedures in health and social care IT systems; ${ }^{10}$ they have been in use in the NHS since $1985 .{ }^{10}$ A drug dictionary based on data from the Multilex classification has been used to code drugs in the database. ${ }^{7}$ Successive updates of patients' records to the database include any subsequent changes made by GPs.

The study population comprised a randomised sample of 100 matched patients from the THIN database, who were treated with either CMC, DM, F, $\mathrm{K}$ or S, during 2011. Patients were included in the study, provided they met the following criteria:

$-\geq 18$ years of age.

- VLU duration $\geq 3$ months, before receiving their first treatment with one of the study dressings

- Not received treatment for their VLU with any of this study's comparators within 6 months of starting treatment with one of the study dressings

- Had at least 6 months' follow-up data in their case record, following the start of treatment.

Patients in each group were matched according to age, gender, their general practice, date of diagnosis of their VLU and treatment start date. One patient in each of the $\mathrm{CMC}, \mathrm{F}, \mathrm{K}$ and $\mathrm{S}$ group had to be excluded, as they did not meet the inclusion criteria, generating a final sample size of 99 patients per group. Fifty-seven patients in the DM group had to be excluded because of underlying skin cancer, generating a final sample size of 43 patients.

\section{Ethics approval}

Ethics approval to use patients' records from the THIN database for this study was obtained from a Research Ethics Committee that appraises studies using the THIN database.

\section{Study variables}

Information extracted from patients' records included age, gender, symptoms, comorbidities and duration of symptoms. Community-based and secondary care VLU-related resource use, over a period of 6 months following the treatment start date, was also extracted. Specific wound size estimates for all patients were not available for every dressing change. In those instances, and based on previous experience, ${ }^{11}$ patients' wound size was assumed to be $80 \%$ of the size of the primary dressing.

Patients' outcomes and resource use were quantified for all five groups. Differences between groups were tested for statistical significance using a MannWhitney U test or Chi-squared test. Logistic regression was performed to identify independent predictors of healing. Multiple, ordinary least-squares regression was also performed to assess the impact of the wounds' baseline characteristics on resource use and clinical outcomes.

\section{Health economic modelling}

A decision model was constructed depicting the patient pathways and associated management of the cohort of patients in each of the five groups, using patient level data. The model was populated with probabilities, health-care resource utilisation and clinical outcomes extracted from the THIN data set. The model spans a period of 6 months following the start of treatment with each dressing and comprises the following health states: unchanged ulcer, improved ulcer, worsened ulcer, healed ulcer and death. It was decided that since the time frame was relatively short and recurring events (in terms of patients moving into different health states) were relatively static, a decision model was the most appropriate type to address the study objectives.

Utility scores express patient preferences for specific health states on a scale ranging from 0 (representing death) to 1 (representing perfect health). These scores provide the weights to estimate healthrelated quality of life, in terms of the number of quality-adjusted life years (QALYs) gained by an intervention or service. Health-related quality of life 
Table I. Patients' characteristics in the dataset, at study start

\begin{tabular}{|c|c|c|c|c|c|}
\hline & CMC & DM & $\mathbf{F}$ & $\mathbf{K}$ & $\mathbf{S}$ \\
\hline No. of patients & 99 & 43 & 99 & 99 & 99 \\
\hline Age (years)* & $\begin{array}{l}74.3 \\
(71.5 ; 77.0)\end{array}$ & $\begin{array}{l}71.7 \\
(67.1 ; 76.3)\end{array}$ & $\begin{array}{l}74.9 \\
(72.6 ; 77.3)\end{array}$ & $\begin{array}{l}70.3 \\
(67.1 ; 73.4)\end{array}$ & $\begin{array}{l}74.3 \\
(71.6 ; 77.1)\end{array}$ \\
\hline Male (\%) & $52 \%$ & $42 \%$ & $47 \%$ & $47 \%$ & $40 \%$ \\
\hline $\begin{array}{l}\text { Wound duration } \\
\text { (months)*t }\end{array}$ & $\begin{array}{l}3.5 \\
(2.4 ; 4.6)\end{array}$ & $\begin{array}{l}6.8 \\
(5.8 ; 7.8)\end{array}$ & $\begin{array}{l}6.5 \\
(3.9 ; 9.0)\end{array}$ & $\begin{array}{l}9.9 \\
(6.8 ; 13.0)\end{array}$ & $\begin{array}{l}19.8 \\
(14.4 ; 25.3)\end{array}$ \\
\hline $\begin{array}{l}\text { Wound } \\
\text { size }\left(\mathrm{cm}^{2}\right)^{* \neq}\end{array}$ & $\begin{array}{l}62.6 \\
(53.9 ; 71.4)\end{array}$ & $\begin{array}{l}241.9 \\
(190.5 ; 293.3)\end{array}$ & $\begin{array}{l}245.8 \\
(201.9 ; 289.8)\end{array}$ & $\begin{array}{l}277.3 \\
(240.4 ; 3 \mid 4.3)\end{array}$ & $\begin{array}{l}209.7 \\
(177.9 ; 241.6)\end{array}$ \\
\hline $\begin{array}{l}\text { Mean no. } \\
\text { of different } \\
\text { dressings used }\end{array}$ & 1.8 & 2.8 & 2.3 & 2.1 & 3.2 \\
\hline $\begin{array}{l}\text { Analgesic } \\
\text { use }(\%)^{\S}\end{array}$ & $35 \%$ & $49 \%$ & $49 \%$ & $51 \%$ & $46 \%$ \\
\hline $\begin{array}{l}\text { Antibiotic } \\
\text { use (\%) }\end{array}$ & $100 \%$ & $100 \%$ & $100 \%$ & $100 \%$ & $100 \%$ \\
\hline
\end{tabular}

* Results present as mean values with $95 \%$ confidence intervals in parentheses; $\dagger S$ compared with other groups, $p<0.00 I ; \ddagger C M C$ compared with other groups, $p<0.00$; $\S \mathrm{CMC}$ compared with $\mathrm{KM}, \mathrm{p}<0.05$

Table 2. Patient comorbidities

\begin{tabular}{llllll} 
Comorbidities (\%) & CMC & DM & F & K & S \\
\hline Genito-urinary disorder & $19 \%$ & $9 \%$ & $15 \%$ & $9 \%$ & $6 \%$ \\
\hline Endocrinological/metabolic disorder & $33 \%$ & $30 \%$ & $35 \%$ & $34 \%$ & $27 \%$ \\
\hline Cardiovascular disorder & $68 \%$ & $60 \%$ & $73 \%$ & $71 \%$ & $58 \%$ \\
\hline Cerebrovascular disorder & $5 \%$ & $2 \%$ & $2 \%$ & $3 \%$ & $4 \%$ \\
\hline Peripheral vascular disease & $13 \%$ & $5 \%$ & $12 \%$ & $10 \%$ & $16 \%$ \\
\hline Dermatological disorder & $23 \%$ & $21 \%$ & $27 \%$ & $24 \%$ & $20 \%$ \\
\hline Psychiatric illness & $30 \%$ & $53 \%$ & $36 \%$ & $49 \%$ & $30 \%$ \\
\hline Musculoskeletal disorder & $57 \%$ & $56 \%$ & $45 \%$ & $47 \%$ & $48 \%$ \\
\hline Neurological disorder & $10 \%$ & $19 \%$ & $11 \%$ & $16 \%$ & $14 \%$ \\
\hline Pulmonary disease & $14 \%$ & $23 \%$ & $17 \%$ & $20 \%$ & $16 \%$ \\
\hline Immunological disorder & $19 \%$ & $9 \%$ & $24 \%$ & $20 \%$ & $22 \%$ \\
\hline Gastrointestinal disorder & $15 \%$ & $9 \%$ & $18 \%$ & $4 \%$ & $6 \%$ \\
\hline Cancer & $14 \%$ & $28 \%$ & $20 \%$ & $10 \%$ & $14 \%$
\end{tabular}

was not collected in the THIN database; hence, published utilities for VLUs, ${ }^{12}$ previously obtained from the general public across the UK, using standard gamble methodology, were assigned to each patient in the model. This enabled an estimation of patients' expected health status in terms of the number of QALYs at 6 months from the start of treatment with each dressing.

- Model outputs The primary measure of clinical effectiveness used was patients' heath status in terms of the number of QALYs at 6 months. Secondary measures were a range of clinical outcomes, including the probability of healing, time to healing, and reduction in analgesic and antibiotic use over the study period. By assigning unit resource costs at $2010 / 11$ prices $^{13-15}$ to the resource utilisation estimates within the decision model, the 6-monthly health-care cost of managing patients treated with each dressing was estimated.

- Cost-effectiveness analyses The cost-effectiveness of the different dressings relative to one another was calculated as the difference between the cost of alternative treatment strategies over 6 months, divided by the difference in the number of QALYs between the same alternative treatment strategies at 6 months. Hence, the cost-effectiveness of one dressing relative to another was defined as the cost per QALY gained. If one of the dressings resulted in an improved outcome for less cost, it was considered to be the dominant treatment.

- Sensitivity analyses To assess uncertainty within the model, bootstrapping was undertaken to estimate the distribution of expected costs, outcomes and cost-effectiveness ratios. This involved generating 10000 subsets of the data from each group on the basis of random sampling and replacing the data once sampled. Use of these subsets enabled the construction of cost-effectiveness acceptability curves, showing the probability of alternative treatments being cost-effective at different thresholds. In order to rank the superabsorbents in order of value for money to the NHS, the net monetary benefit of each was generated from the boostrapped subsets. Additionally, deterministic sensitivity analyses were performed on all the model's inputs to identify how the incremental cost-effectiveness of one strategy over the other would change by varying the different parameters in the model.

\section{Ranking of attributes}

In a study by Vermeulen et al., ${ }^{16}$ different attributes of wound dressings were scored as follows:

- Frequency of dressing change $=14$

- Pain during dressing change $=7$

- Wound healing time $=5$

The importance of the attributes being reflected by the higher score. Healing was not ranked in this study; however, we assumed it to be as important as frequency of dressing change, and assigned it a value of 14. Additionally, reduction in analgesic use was used as a proxy for pain during dressing change. 
The superabsorbents were ranked from 4 to 1 in order of decreasing value for each of these attributes individually. This value was then multiplied by the score estimated by Vermeulen et al. in order to value and rank the superabsorbents, according to their attributes.

\section{Results}

Patients in the five groups were matched by age and gender (Table 1), with no significant differences in patients' comorbidities detected between the groups, using a Chi-squared test (Table 2). However, patients treated with $\mathrm{S}$ had significantly older wounds than patients in the other four groups. Nevertheless, patients only received a mean 2-3 different dressings before the start of their study treatment, irrespective of the age of their wound (Table 1). Additionally, the wounds in the CMC group were significantly smaller than those in the other four groups (Table 1).

Approximately a third of patients in all five groups were initially treated at the time of diagnosis with an antimicrobial dressing; $14-27 \%$ of patients were treated with a soft polymer dressing and 10-17\% with an absorbent dressing (but not CMC). None of the patients received another superabsorbent before the start of their study treatment. Patients' use of dressings before the start of their study treatment is summarised in Table 3.

\section{Patient management and outcomes}

Between $17 \%$ and $23 \%$ of patients never received compression for their VLU. Moreover, no significant differences were observed in the wound healing rate and time to healing between the five groups. However, there was a significant difference in the percent change in size of unhealed wounds in the CMC group compared with the other four groups. Wounds that remained unhealed in the CMC group increased in size by a mean $43 \%$, whereas the unhealed wounds in the other groups decreased in size by $20-53 \%$ $(\mathrm{p}<0.001)$. There were no differences in the wound size at baseline between those wounds that healed and those that remained unhealed, in each group, except the $\mathrm{F}$ group. The wounds that remained unhealed in the F group were larger than the wounds that healed. Analgesic and antibiotic use decreased during the study period and $<5 \%$ of patients died. However, there were no significant differences in patients health status in terms of their number of QALYs at 6 months (Table 4).

Logistic regression showed that the age of the wound was an independent predictor of non-healing (OR: 0.982 [95\%CI: 0.968; 0.993]; for each month; $\mathrm{p}<0.02$ ). Additionally, analgesic use at the start of treatment was an independent predictor of non-healing (OR: 0.622 [95\%CI: 0.453; 0.886]; $\mathrm{p}<0.05)$. However, logistic regression showed that
Table 3. Dressings used before the start of the study treatments

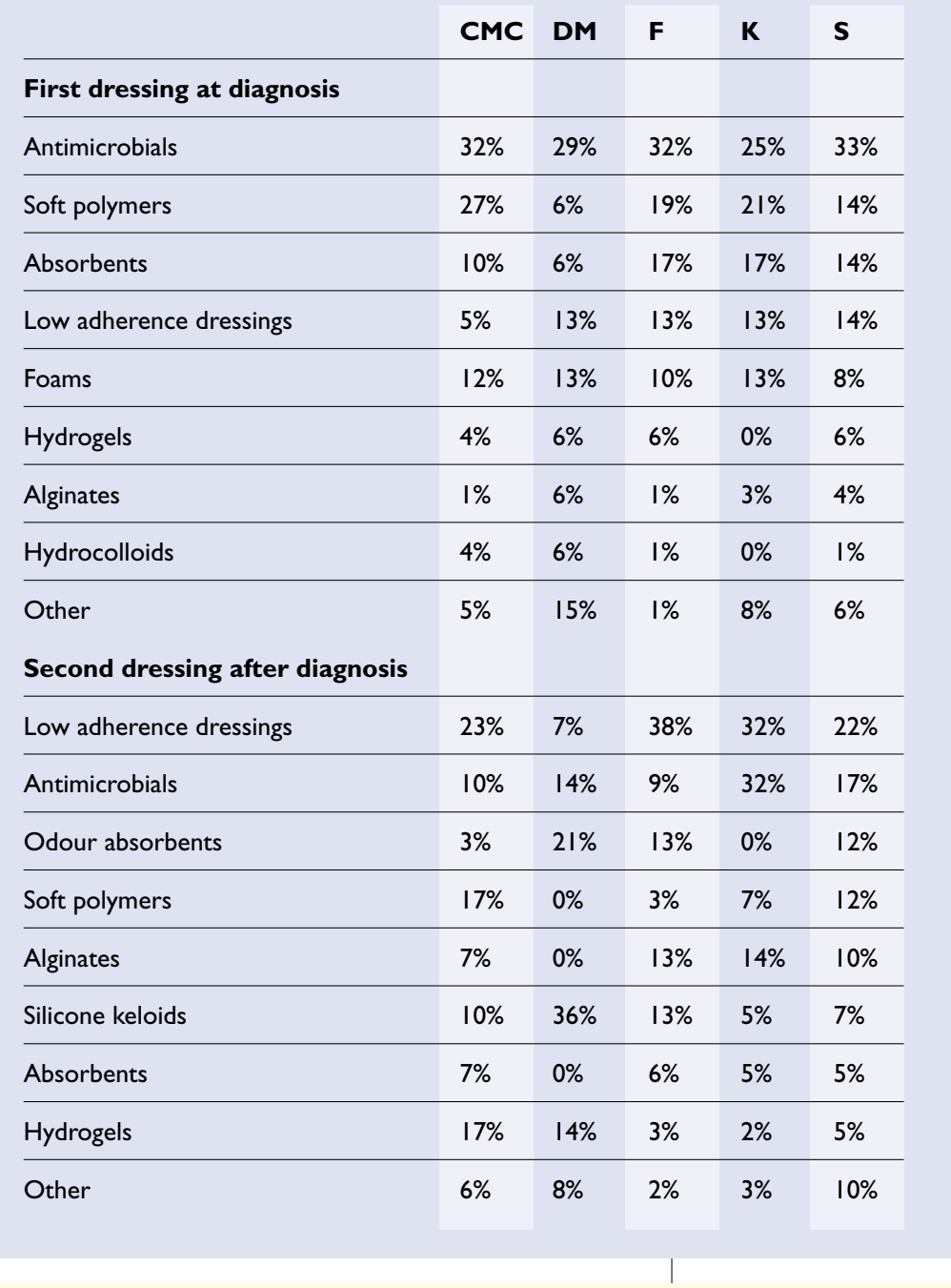

Fig I. Percentage of patients who received an analgesic at the start of treatment

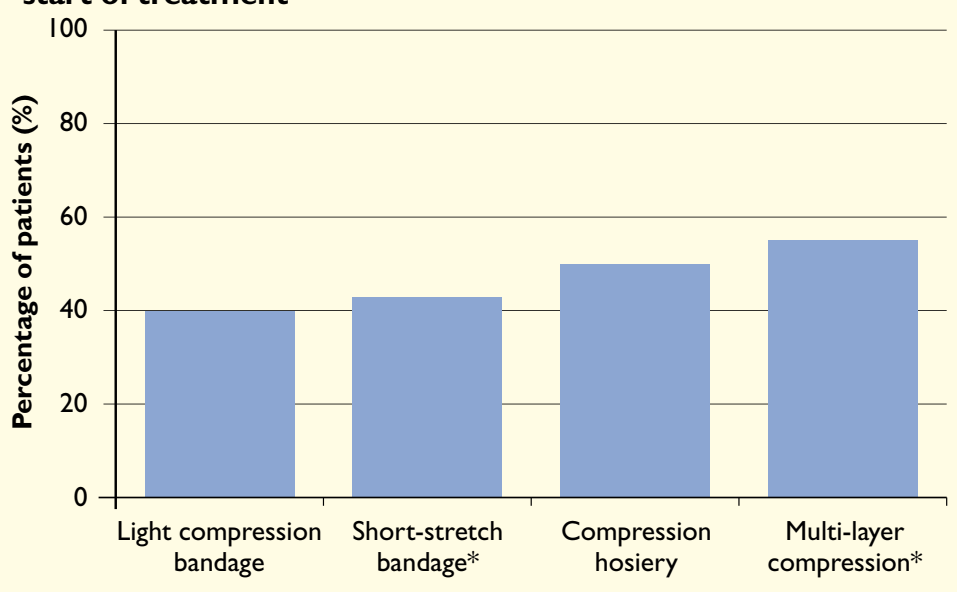

* Short stretch compared with multi-layer, $\mathrm{p}<0.05$ 


\section{References}

I White, R., Cutting, K. Modern exudate management: a review of wound treatments. World Wide Wounds. 2006; Available from: http:// tinyurl.com/6cfloz

[Accessed February 2013].

2 World Union of Wound Healing Societies (WUWHS). Wound exudate and the role of dressings. A consensus document. WUWHS, 2007. Available from: http:// tinyurl.com/8c8ffft

[Accessed February 2013]

3 Dowsett, C., Newton, H. Wound bed preparation: TIME in practice. Wounds UK. 2005; I: 3, 58-70.

4 Bishop, S. Importance of moisture balance at the wound-dressing interface.J Wound Care. 2003; 12 : 125-128.

5 Vuolo, J. Current options for managing problems of excess exudate. Prof Nurse. 2004; 19: 487-490.

6 Stephen-Haynes, J.

Managing exudate and the

key requirements of absorbent dressings. Br J Comm Nurs. 2011; 16:3 (Suppl.), S22-S27.

7 The Health Improvement Network (THIN) Database. London: EPIC, 20II. 8 Hippisley-Cox,.., Coupland, C.,Vinogradova, Y. et al. Performance of the QRISK cardiovascular risk prediction algorithm in an independent UK sample of patients from general practice: a validation study. Heart. 2008; 94: 34-39.

9 Blak, B.T.,Thompson, M., Dattani, H., Bourke,A. Generalisablity of The Health Improvement Network (THIN) database: demographics, chronic disease prevalence and mortality rates. Inform Prim Care. 201I; 19:25I-255. continued on page $1 / 8$

Table 4. Patient outcomes at 6 months

\begin{tabular}{llllll} 
& CMC & DM & F & K & S \\
\hline Changed superabsorbent (\%) & $7 \%$ & $28 \%$ & $25 \%$ & $8 \%$ & $16 \%$ \\
\hline Never received compression (\%) & $23 \%$ & $23 \%$ & $17 \%$ & $19 \%$ & $18 \%$ \\
\hline Wound healed (\%) & $56 \%$ & $39 \%$ & $45 \%$ & $54 \%$ & $55 \%$ \\
\hline Change in size of unhealed wounds (\%) & $43 \%$ & $-22 \%$ & $-53 \%$ & $-20 \%$ & $-40 \%$ \\
\hline Time to healing (months)* & $2.6(2.3 ; 3.0)$ & $2.1(1.6 ; 2.6)$ & $3.3(3.0 ; 3.6)$ & $2.8(2.4 ; 3.2)$ & $2.9(2.6 ; 3.2)$ \\
\hline Mean size of wound that healed $\left(\mathrm{cm}^{2}\right)^{*}$ & $\begin{array}{l}58.0 \\
(30.2 ; 51.5)\end{array}$ & $\begin{array}{l}(147.5 ; 256.5) \\
(124.9 ; 228.0)\end{array}$ & $\begin{array}{l}(142.8 ; 245.3) \\
(133.2 ; 232.6)\end{array}$ \\
\hline Mean size of wound that remained & 68.9 & 206.0 & 284.1 & 265.1 & 196.4 \\
unhealed (cm²)* & $(34.0 ; 63.5)$ & $(146.3 ; 265.7)$ & $(189.6 ; 337.1)$ & $(125.1 ; 237.5)$ & $(93.0 ; 171.1)$ \\
\hline Reduction in analgesic use over study (\%) & $22 \%$ & $47 \%$ & $27 \%$ & $11 \%$ & $31 \%$ \\
\hline Reduction in antibiotic use over study $(\%)^{5}$ & $34 \%$ & $19 \%$ & $22 \%$ & $27 \%$ & $32 \%$ \\
\hline No. of QALY* & 0.367 & 0.335 & 0.343 & 0.344 & 0.345 \\
\hline Died (\%) & $(0.365 ; 0.369)$ & $(0.332 ; 0.338)$ & $(0.341 ; 0.345)$ & $(0.342 ; 0.346)$ & $(0.343 ; 0.347)$ \\
\hline & $2 \%$ & $5 \%$ & $5 \%$ & $1 \%$ & $3 \%$
\end{tabular}

*Values in parenthesis represent $95 \%$ confidence intervals;

$+C M C$ compared with other groups, $p<0.001 ; \neq D M$ compared with other groups, $p<0.02 ; \S D M$ compared with other groups, $p<0.05$

patients' age, gender, initial wound size and type of absorbent dressing used were not independent predictors of healing.

Logistic regression also showed that use of compression was an independent predictor of patients using analgesics over the study period (OR: 2.842 [95\%CI: 1.63; 5.68]; $\mathrm{p}<0.01$ ), while use of antibiotics during the study period was also an independent predictor of patients using analgesics (OR: 3.244 [95\%CI: $1.855 ; 5.698] ; \mathrm{p}<0.001)$. Use of analgesics was not affected by patient's age, gender, initial wound size, wound age or absorbent dressing used. However, use of analgesics at the start of treatment varied according to the compression system used (Fig 1).

Use of compression and wound age were both independent predictors of patients using antibiotics over the study period: use of compression (OR: 1.88 (95\%CI:1.15; 3.14); $\mathrm{p}<0.03$ ) and wound age (OR: 1.021 (95\%CI: 0.990; 1.039$)$ for each month; $\mathrm{p}<0.02$ ). Use of analgesics during the study period was an independent predictor of patients using antibiotics (OR: 3.263 (95\%CI: $1.929 ; 5.595) ; \mathrm{p}<0.001)$. However, use of antibiotics was not affected by a patient's age, gender, initial wound size or absorbent dressing used.

\section{Resource use}

There were differences in resource use between the five groups, although they were not statistically significance (Table 5). Patients were predominantly managed by practice nurses and community nurses. Only three patients were seen by a tissue viability nurse for one visit in a hospital outpatient clinic. Thirty-five per cent of all patients were seen by a GP for their VLU and 23\% were referred to a physician in an outpatient clinic. Patients' dressings were changed, on average, every $2-4$ days and, over the 6-month study period, patients received a mean 2-3 dressings and a compression bandage at each dressing change.

Multiple linear regression showed that the 6-monthly number of nurse visits was affected by:

- Use of compression, which was associated with 8.2 more nurse visits $(\mathrm{p}<0.001)$

- Use of analgesics at the start of treatment, which was associated with 5.2 more nurse visits $(\mathrm{p}<0.005)$

- Patients' age, which was associated with 1.3 fewer nurse visits for each 10 years of age $(\mathrm{p}<0.05)$

- Wound age, which was associated with 1.0 more nurse visit for each 10 months $(\mathrm{p}<0.02)$

- Wound size at the start of treatment, which was associated with 1.0 more nurse visit for each $100 \mathrm{~cm}^{2}(\mathrm{p}<0.05)$.

\section{Health-care cost of patient management}

The 6-monthly NHS cost of using each of the dressings to manage a patient with a VLU is summarised in Table 6. The 6-monthly NHS cost of managing a VLU with S was $£ 3700$ per patient, which was $15-28 \%$ lower than the cost of managing patients with the other three superabsorbents (Table 6). Practice and community nurse visits were the primary cost drivers in all groups, accounting for $67-78 \%$ of the 6-monthly NHS cost. Dressings accounted for up to a further $22 \%$ of the costs and compression bandages for up to a further 7\%. GP visits and hospital outpatient visits accounted for $<3 \%$ of the NHS cost of patient management. 
Table 5. Mean 6-monthly amount of resource use per patient

\begin{tabular}{|c|c|c|c|c|c|}
\hline Resource & CMC & DM & $\mathbf{F}$ & $\mathbf{K}$ & $\mathbf{S}$ \\
\hline GP surgery visits* & $0.6(0.4 ; 0.8)$ & $0.7(0.4 ; 1.0)$ & $0.6(0.4 ; 0.8)$ & $0.4(0.2 ; 0.6)$ & $0.5(0.3 ; 0.7)$ \\
\hline GP home visits* & $0.1(0.0 ; 0.2)$ & $0.1(0.0 ; 0.2)$ & $0.2(0.0 ; 0.4)$ & $0.1(0.0 ; 0.2)$ & $<0.1(0.0 ; 0.1)$ \\
\hline Practice nurse visits* & I8.I $(14.2 ; 22.0)$ & $19.0(12.8 ; 25.3)$ & $14.4(|1.6 ;| 7.2)$ & $19.5(14.9 ; 24.1)$ & $16.0(12.9 ; 19.2)$ \\
\hline Community nurse visits* & $22.5(17.6 ; 25.4)$ & $38.6(25.9 ; 51.3)$ & $67.9(54.5 ; 81.3)$ & $54.1(41.3 ; 66.9)$ & $43.9(35.3 ; 52.6)$ \\
\hline Hospital outpatient visits* & $0.6(0.3 ; 0.9)$ & $0.7(0.2 ; 1.2)$ & $0.6(0.4 ; 0.8)$ & $0.9(0.6 ; 1.3)$ & $0.5(0.2 ; 0.8)$ \\
\hline No. of dressings* & $\begin{array}{l}117.0 \\
(94.0 ; 140.1)\end{array}$ & $\begin{array}{l}171.4 \\
(104.8 ; 238.0)\end{array}$ & $\begin{array}{l}183.6 \\
(140.2 ; 227.0)\end{array}$ & $\begin{array}{l}215.3 \\
(155.9 ; 274.7)\end{array}$ & $\begin{array}{l}131.9 \\
(92.9 ; 170.9)\end{array}$ \\
\hline No. of compression bandages* & $\begin{array}{l}50.0 \\
(39.2 ; 60.8)\end{array}$ & $\begin{array}{l}57.9 \\
(31.9 ; 83.9)\end{array}$ & $\begin{array}{l}117.3 \\
(80.3 ; 154.3)\end{array}$ & $\begin{array}{l}96.8 \\
(72.0 ; 121.6)\end{array}$ & $\begin{array}{l}83.7 \\
(50.7 ; 116.7)\end{array}$ \\
\hline Laboratory tests* & $0.5(0.3 ; 0.7)$ & $0.3(0.1 ; 0.5)$ & $0.3(0.2 ; 0.4)$ & $0.6(0.3 ; 0.9)$ & $0.3(0.2 ; 0.4)$ \\
\hline Diagnostic procedures* & $0.1(0.0 ; 0.2)$ & $0.1(0.0 ; 0.3)$ & $0.1(0.0 ; 0.2)$ & $0.1(0.0 ; 0.2)$ & $<0.1(0.0 ; 0.1)$ \\
\hline Surgical and therapeutic procedures* & $<0.1(0.0 ; 0.1)$ & $<0.1(0.0 ; 0.1)$ & $<0.1(0.0 ; 0.1)$ & $<0.1(0.0 ; 0.1)$ & $<0.1(0.0 ; 0.1)$ \\
\hline Frequency of dressing change (days) & 4.0 & 3.2 & 2.2 & 2.6 & 3.2 \\
\hline No. of dressings per change & 2.5 & 2.9 & 2.2 & 3.1 & 2.2 \\
\hline No. of bandages per change & 1.0 & 1.0 & 1.4 & 1.3 & 1.3 \\
\hline
\end{tabular}

\section{Cost-effectiveness analyses}

Unhealed wounds that were treated with CMC became larger. Since a clinician cannot predict which wound will heal with any given dressing, and based on the evidence from this study's data set, CMC cannot be recommended for the treatment of highly exuding chronic wounds of $\geq 3$ months of age. Consequently, CMC was excluded from the cost-effectiveness analysis.

Of the other dressings, use of $S$ resulted in a lower 6-monthly NHS cost and more QALYs than the other three superabsorbents. Hence, starting treatment with $\mathrm{S}$ was found to be the dominant strategy. Starting treatment with $\mathrm{F}$ instead of DM resulted in a NHS cost increase of $£ 787$ and a gain of 0.01 QALYs, and starting treatment with $\mathrm{K}$ instead of DM resulted in a NHS cost increase of $£ 422$ and a gain of 0.01 QALYs. Hence, the cost per QALY gained of using $\mathrm{K}$ or $\mathrm{F}$ instead of DM was $£ 50376$ and $£ 106935$, respectively. Hence, neither K nor F are cost-effective relative to DM, as the cost per QALY gained is greater than the National Institute for Health and Clinical Excellence's (NICE) recommended threshold of $£ 20000 .{ }^{17}$

These analyses suggest that $S$ is the preferred treatment followed by $\mathrm{DM}, \mathrm{K}, \mathrm{F}$ and $\mathrm{CMC}$, in that order. Notwithstanding this, neither $\mathrm{K}$ nor $\mathrm{F}$ are expected to afford the NHS value for money, when compared with S and DM.

\section{Sensitivity analyses}

Bootstrapping was performed to identify the distribution in the incremental costs and QALYs at
Fig 2. Scatterplot of the incremental cost-effectiveness of $S, K$ and F, each compared with DM at 6-months after start of treatment

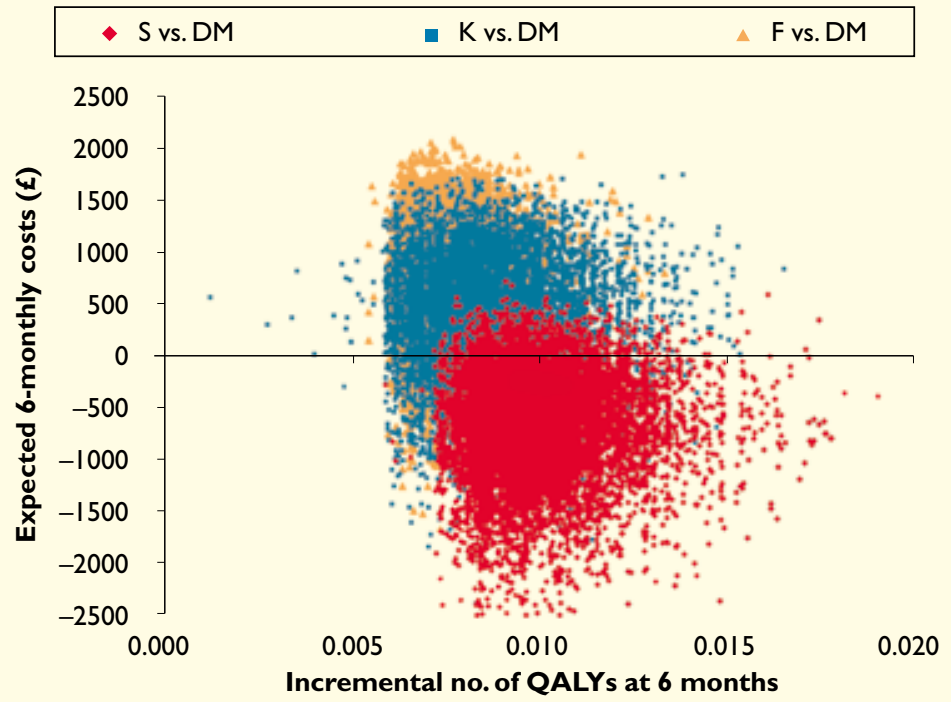

6 months, for the four alternative superabsorbents (Fig 2), with samples from the more cost-effective dressings tending towards the bottom right-hand (dominant) quadrant.

Cost-effectiveness acceptability curves were generated from the boostrapped subsets (Fig 3). These demonstrated that, at a cost-effectiveness threshold of $£ 20000$ per QALY, up to $97 \%$ of a cohort is expected to be treated cost-effectively with $\mathrm{S}$ compared with 
Table 6. Mean 6-monthly NHS cost of resource use per patient (at 2010/I I prices)

\begin{tabular}{|c|c|c|c|c|c|}
\hline Resource & CMC & DM & $\mathbf{F}$ & $\mathbf{K}$ & $\mathbf{S}$ \\
\hline GP visits & $29.9(1 \%)$ & $26.2(1 \%)$ & $45.5(1 \%)$ & $24.2(1 \%)$ & $18.2(<1 \%)$ \\
\hline Practice nurse visits & 819.7 (33\%) & ) $946.4(22 \%)$ & $696.5(14 \%)$ & 916.4 (19\%) & $715.3(19 \%)$ \\
\hline $\begin{array}{l}\text { Community nurse } \\
\text { visits }\end{array}$ & $\begin{array}{l}1023.2 \\
(42 \%)\end{array}$ & $\begin{array}{l}1983.1 \\
(46 \%)\end{array}$ & $\begin{array}{l}3292.4 \\
(64 \%)\end{array}$ & $\begin{array}{l}2535.7 \\
(53 \%)\end{array}$ & $\begin{array}{l}1958.4 \\
(53 \%)\end{array}$ \\
\hline Outpatient visits & $55.6(2 \%)$ & $73.4(2 \%)$ & $61.6(1 \%)$ & $79.3(2 \%)$ & $48.6(1 \%)$ \\
\hline Dressings & 259.3 (11\%) & $963.6(22 \%)$ & ) $653.1(13 \%)$ & $534.7(11 \%)$ & $458.8(12 \%)$ \\
\hline $\begin{array}{l}\text { Compression } \\
\text { bandages }\end{array}$ & $136.0(6 \%)$ & $193.4(4 \%)$ & $274.1(5 \%)$ & $290.3(6 \%)$ & 259.1 (7\%) \\
\hline Drugs & $53.3(2 \%)$ & $97.3(2 \%)$ & $70.8(1 \%)$ & I23.I (3\%) & $79.8(2 \%)$ \\
\hline $\begin{array}{l}\text { Laboratory/ } \\
\text { diagnostic tests }\end{array}$ & $6.0(<1 \%)$ & $5.3(<1 \%)$ & $3.6(<1 \%)$ & $3.3(<1 \%)$ & $1.0(<1 \%)$ \\
\hline $\begin{array}{l}\text { Surgical/therapeutic } \\
\text { procedures }\end{array}$ & $54.0(2 \%)$ & $0.0(0 \%)$ & $16.0(<1 \%)$ & $241.0(5 \%)$ & $90.0(2 \%)$ \\
\hline Consumables & $15.8(1 \%)$ & $58.0(1 \%)$ & $14.0(<1 \%)$ & $20.1(<1 \%)$ & $45.1(1 \%)$ \\
\hline Total & 2452.8 & 4346.7 & 5127.6 & 4768.1 & 3674.3 \\
\hline
\end{tabular}

Percentage of total cost is given in parentheses

Table 7. Sensitivity analyses showing the range in the mean 6-monthly NHS cost, and number of QALYs per patient, associated with varying different parameters in the model

\begin{tabular}{|c|c|c|c|c|}
\hline Scenario & $\mathbf{S}$ & $\mathbf{K}$ & $\mathbf{F}$ & DM \\
\hline $\begin{array}{l}\text { Healing rate ranges } \\
\text { from } 0.25 \text { to } 0.75\end{array}$ & $\begin{array}{l}\qquad 79 \\
(0.1\end{array}$ & $\begin{array}{l}£ 10326-£ 3442 \\
(0.159-0.476)\end{array}$ & $\begin{array}{r}2 £ 9333-£ 3111 \\
(0.189-0.566)\end{array}$ & $\begin{array}{l}£ 6840-2280 \\
(0.213-0.639)\end{array}$ \\
\hline
\end{tabular}

Time to healing ranges $€ 7348-£ 2449 £ 9538-£ 3179 \quad £ 10266-£ 3422 £ 4346-4346$ from $50 \%$ below to $50 \%(0.533-0.283)(0.530-0.282) \quad(0.499-0.291) \quad(0.467-0.291)$ above base case values

No. of practice nurse $\quad € 3317-£ 4032 £ 4310-£ 5226 \quad £ 4779-£ 5476 \quad £ 3874-£ 4820$ $\begin{array}{lllll}\text { visits ranges from } 50 \% & \text { (No effect) (No effect) (No effect) (No effect) }\end{array}$ below to $50 \%$ above

base case values

No. of community nurse $£ 2695-£ 4654 £ 3500-£ 6036 \quad £ 3481-£ 6774 \quad £ 3355-£ 5338$ $\begin{array}{llllll}\text { visits ranges from } 50 \% & \text { (No effect) } & \text { (No effect) } & \text { (No effect) } & \text { (No effect) }\end{array}$ below to $50 \%$ above

base case values

No. of dressings ranges $€ 3445-£ 3904 £ 450 \mathrm{I}-£ 5035 \quad £ 480 \mathrm{I}-£ 5454 \quad £ 3865-£ 4829$ from $50 \%$ below to $50 \%$ (No effect) (No effect) (No effect) (No effect) above base case values

No. of compression $\quad € 3545-£ 3804 £ 4623-£ 4913 \quad £ 4991-£ 5265 \quad € 4250-£ 4443$ $\begin{array}{llll}\text { bandages ranges from (No effect) (No effect) (No effect) (No effect) } & \end{array}$ $50 \%$ below to $50 \%$

above base case values

Range in mean 6-monthly number of QALYs per patient given in parentheses
DM. However, only $30 \%$ of a cohort is expected to be treated cost-effectively with $\mathrm{K}$ compared with DM and only $10 \%$ is expected to be cost-effectively treated with F compared to DM. These analyses suggest that neither K or F would afford a cost-effective use of NHS resources when compared with $\mathrm{S}$ and DM.

Analysis of the net monetary benefit of each superabsorbent demonstrated that $\mathrm{S}$ affords the greatest value for money to the NHS, followed by $\mathrm{DM}, \mathrm{K}$ and $\mathrm{F}$, implying that $\mathrm{S}$ should be the preferred treatment of choice and $\mathrm{F}$ should be the last treatment of choice (Fig 4).

Deterministic sensitivity analyses were performed on all the model's inputs, but only the main findings have been presented (Table 7). These analyses found the relative cost-effectiveness of $\mathrm{S}, \mathrm{DM}, \mathrm{K}$ and $\mathrm{F}$ to be sensitive to changes in healing rates, time to healing, number of practice nurse visits, number of community nurse visits and number of dressings. The relative cost-effectiveness of the four superabsorbents was not sensitive to changes in any other model input.

\section{Ranking of attributes}

When the score estimated by Vermeulen et al. was assigned to each of the superabsorbents, it was found that $\mathrm{S}$ scored the highest value followed by $\mathrm{DM}, \mathrm{K}$ and the $\mathrm{F}$ (Table 8 ). This ranking was consistent with that identified by the estimated net monetary benefit of each of the superabsorbents and reinforces the finding that $S$ is the preferred dressing and $\mathrm{F}$ should be the last treatment of choice. This may be indicative of the finding that the ability of $\mathrm{F}$ to facilitate healing may be constrained by wound size, whereas there was no difference in wound size between those wounds that did and did not heal with the other three superabsorbents.

\section{Discussion}

This comparative study aimed to determine the relative clinical and cost-effectiveness of using CMC, $\mathrm{DM}, \mathrm{F}, \mathrm{K}$ and $\mathrm{S}$ in the treatment of highly exuding chronic VLUs in the UK. Accordingly, patients in the THIN database who had a VLU that was $\geq 3$ months of age and who were initially managed with one of these dressings were compared and followed-up for 6 months. The advantage of using the THIN database is that the treatment patterns and associated resource use observed in this study were based on actual clinical practice, rather than trial protocol-driven resource use.

However, this naturalistic approach does have its limitations. Patients were not randomised to the treatment they received and resource use, while collected prospectively, was analysed retrospectively. Moreover, DM was used to a greater extent to treat leg ulcers with an underlying cancer aetiology than the other superabsorbents. These patients were excluded from the analysis, as it was considered that 
the outcomes of managing these wounds would be different to that of routine management of venous leg ulcers and not be consistent with the other wounds analysed in this study.

Consequently, 439 matched patients were included in the analysis. It is notoriously difficult to power health economic studies where the metric is use of different resources or a range of clinical outcomes, which are unknown at the outset. However, power calculations showed that the sample size was sufficiently large to detect any significant differences in nurse visits with 95\% power and a type I (alpha) error of 0.05 between the groups, had they occurred.

For patients to have been included in the data set they had to have received CMC, DM, F, K or S for their VLU, and be matched on the basis of their age, gender, the same general practice and date of diagnosis of their VLU. However, the size of the VLUs treated with CMC were significantly smaller than those in the other four groups. Additionally, wounds treated with S were significantly older than those in the other four groups. Nevertheless, regression analysis showed that healing was not affected by the wound size at the start of treatment. A wound's age at the start of treatment was an independent predictor of non-healing; however, due to the nature of the data set, it was not possible to assess whether the healing rate associated with $\mathrm{S}$ would have been higher if used to treat wounds of 6 months rather than 20 months of age.

The findings raise the question as to why $S$ was reserved for wounds that were significantly older than the wounds treated with the other dressings. However, irrespective of a wound's age, the patients in the data set only received $2-3$ different dressings before the start of the study treatment and, interestingly, none of the patients received another superabsorbent before the start of their study treatment.

The patients in the data set were managed by a practice or community nurse, who saw them on average every 2-4 days. Moreover, only 35\% of all patients were seen by a GP for their VLU and only $23 \%$ were referred to a hospital specialist. Additionally, only three patients were seen by a tissue viability nurse for one appointment. This could be a reflection of how most patients with a VLU are now being managed in the community in the UK. The VLU healing rates found in this study, which reflect 'real-world' practice, are consistent with those reported in other studies (55-65\% at 12 weeks). ${ }^{18,19}$

Our study does not address the question of who should be diagnosing and managing VLUs, how should it be done or the more complex question of integrated care pathways. However, in the NHS' Venous Leg Ulcer and Wound Healing Implementation Pack, a healing rate below 70\% at 6 months and an infection rate of $>0 \%$ would be deemed a breach of performance. ${ }^{20}$ Consequently, implementation of
Table 8. Ranking of attributes

Ranking of attributes based on this study's findings

\begin{tabular}{|c|c|c|c|c|}
\hline & DM & $\mathbf{F}$ & $\mathbf{K}$ & $\mathbf{s}$ \\
\hline Healing & I & 2 & 3 & 4 \\
\hline Time to healing & 4 & I & 3 & 2 \\
\hline Reduction in analgesic use & 4 & 2 & I & 3 \\
\hline Frequency of dressing change & 4 & I & 2 & 4 \\
\hline
\end{tabular}

Value of attributes using the ranking value estimated by Vermeulen et al.

\begin{tabular}{l|l|l|l|l}
\hline Healing & 14 & 28 & 42 & 56 \\
\hline Time to healing & 20 & 5 & 15 & 10 \\
\hline Reduction in analgesic use & 28 & 14 & 7 & 21 \\
\hline Frequency of dressing change & 56 & 14 & 28 & 56 \\
\hline Total value of attributes & 118 & 61 & 92 & 143 \\
\hline
\end{tabular}

Fig 3. Cost-effectiveness acceptability curves

\begin{tabular}{|lll|}
\hline S vs.DM $\quad$ Kvs.DM $\quad$ Fvs.DM \\
\hline
\end{tabular}

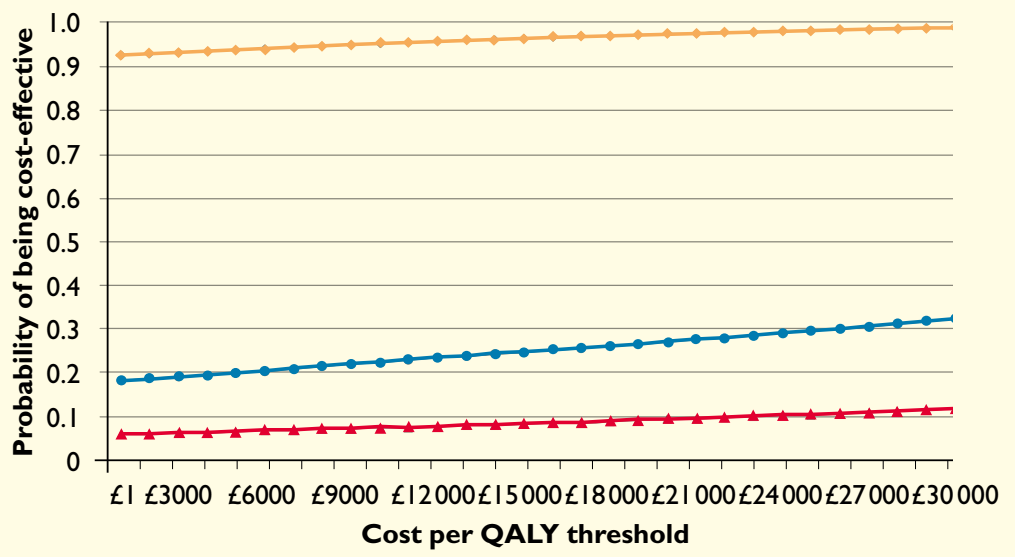

care pathways, improving clinician education in primary care on the management of VLUs has the potential to increase the use of cost-effective interventions, improve outcomes and release health-care resources for alternative use within the system.

\section{Limitations}

This study has a number of other limitations. The results were censored at 6 months and excluded the costs and consequences of managing patients beyond this period. The THIN database may have under-recorded use of some health-care resources outside the GP's surgery, such as home visits made by GPs, home visits made by nurses, outpatient 
Fig 4. Net monetary benefit of the four superabsorbents

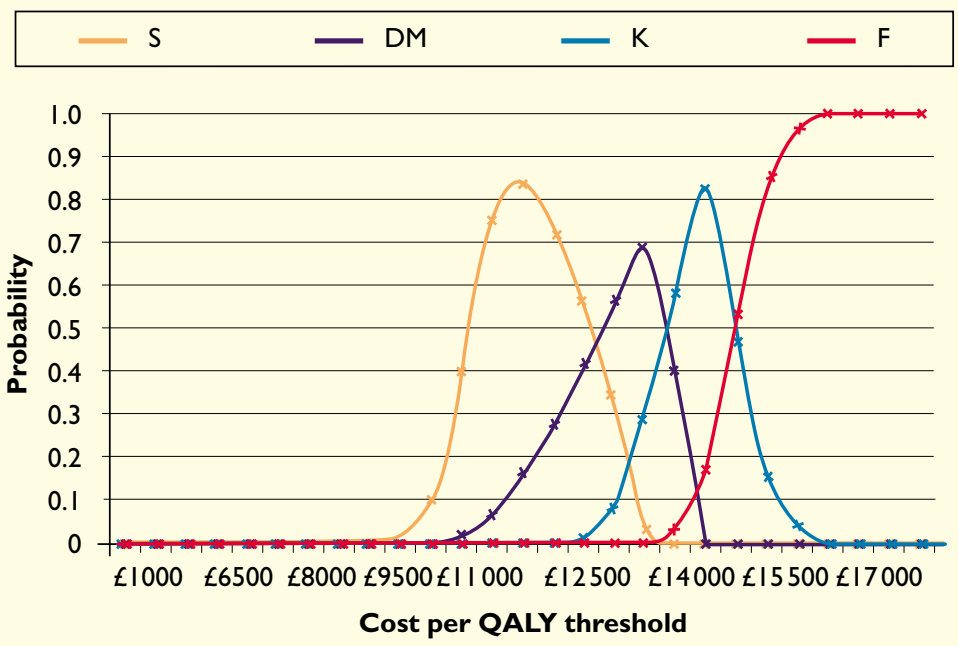

visits, and attendances at accident and emergency departments. The analysis only considered the cost of NHS resource use for the 'average patient' and no attempt was made to stratify resource use and costs according to gender, comorbidities, suitability of patients for different treatments and other diseaserelated factors. Also excluded were the costs incurred by patients and indirect costs incurred by society as a result of patients taking time off work. However, the patients' mean age was $>65$ years, so it is unlikely that many were in employment.

There are many outcomes that could be used to assess the effect of an intervention on a wound, including wound healing, time to healing, wound size reduction, granulation, exudate reduction and epithelialisation. However, the records in the THIN database did not include all of this information for all patients. In particular, the reasons why unhealed wounds in the CMC group increased in size. This might be due to maceration, but the findings warrant investigation in a prospective study. Notwithstanding these limitations, $\mathrm{S}$ appears to be clinically more

10 Department for Health (DH). Read Codes. DH, 2010; Available from: http://tinyurl.com/co544tj [Accessed February 2013].

I I Guest, J.F., Taylor, R.R.,Vowden, K., Vowden, P. Relative

cost-effectiveness of a skin

protectant in managing venous leg ulcers in the UK. JWound Care. 2012; 21: 389-398.

12 Clegg, J.P., Guest, J.F. Modelling the cost-utility of bio-electric stimulation therapy (Posifect)

compared to standard care in the treatment of elderly patients with chronic nonhealing wounds in the UK. Curr Med Res Opin. 2007; 23 87I-883.
13 Department of Health (DH) NHS Reference Costs 2010/II; Available from: http:// www.dh. gov.uk [Accessed February 20I3] 14 Curtis, L. Unit Costs of Health and Social Care 20I I. University of Kent. Personal Social Services Research Unit, 201I.

15 Drug Tariff 201 I.Available from: http://www.drugtariff.com [Accessed February 2013]. 16 Vermeulen, H., Ubbink, D.T., de Zwart, F. et al. Preferences of patients, doctors, and nurses regarding wound dressing characteristics: a conjoint analysis. Wound Repair Regen. 2007; 15 : 302-307.

effective than the other superabsorbents in treating highly exuding chronic VLUs, as judged by the attributes that could be quantified. Furthermore, use of $\mathrm{S}$ leads to lower NHS costs. Over the 6 months following the start of treatment with S, the NHS cost of VLU management was estimated to be $\sim £ 3800$, which was $15-28 \%$ less than the cost of managing patients with the other superabsorbents evaluated in this study. Unsurprisingly, the overall 6-monthly cost of patient management and the patterns of resource use were concordant with our previous health economic studies on VLU management. ${ }^{11,12,21}$

This evaluation provided an estimate of the clinical outcomes, resource implications and associated costs attributable to managing VLUs with five different dressings. While the study results are compelling, the analyses of clinical outcomes were based on clinicians entries into their patients' records, and inevitably subject to a certain amount of imprecision and lack of detail. Moreover, the computerised information in the THIN database is collected by GPs for clinical care purposes and not for research. Prescriptions issued by GPs and practice nurses are recorded in the database, but it does not specify whether the prescriptions were dispensed or patient compliance with the product. Consequently, this study's findings should provide a framework for randomised controlled trials comparing the use of alternative superabsorbents in the management of VLUs to prospectively measure wound size, infection, healing and other clinical outcome measures, health-related quality of life in combination with cost-effectiveness metrics. Moreover, given that most patients with a VLU are managed in the community, it was legitimate from a health economics point of view to perform this study from the community perspective using the THIN database.

\section{Conclusion}

Within the limitations of the data set, S affords the NHS a cost-effective treatment for managing highly exuding chronic VLUs of $\geq 3$ months of age, compared with DM, F, K and CMC.

I 7 National Institute for Health and Clinical Excellence (NICE) Guide to the methods of technology appraisal. NICE, 2008. Available from: http://www.nice. org.uk. [Accessed February 20I3]. 18 Vowden, K.R., Mason, A.

Wilkinson, D.,Vowden, P.

Comparison of the healing rates and complications of three four-layer bandage regimens. I Wound Care. 2000; 9: 269-272.

19 Vowden, K.R.,Wilkinson, D., Vowden, P.The K-Four bandage system: evaluating its effectiveness on recalcitrant venous leg ulcers. J Wound Care. 200।; 10: 182-184.
20 Department of Health (DH). Extension of Choice of Any Qualified Provider:Venous Leg Ulcer \&Wound Healing Implementation Pack, 20I I. Available from: http://tinyurl.com/ cxulaz6 [Accessed February 2013]. 21 Guest, J.F., Nagy, E., Sladkevicius, E., et al. Modelling the relative cost-effectiveness of amelogenin in non-healing venous leg ulcers. JWound Care. 2009; 18: 216-224. 